\title{
LEVEL CROSSINGS OF A RANDOM POLYNOMIAL WITH HYPERBOLIC ELEMENTS
}

\author{
K. FARAHMAND
}

(Communicated by Richard T. Durrett)

\begin{abstract}
This paper provides an asymptotic estimate for the expected number of $K$-level crossings of a random hyperbolic polynomial $g_{1} \cosh x+$ $g_{2} \cosh 2 x+\cdots+g_{n} \cosh n x$, where $g_{j}(j=1,2, \ldots, n)$ are independent normally distributed random variables with mean zero, variance one and $K$ is any constant independent of $x$. It is shown that the result for $K=0$ remains valid as long as $K \equiv K_{n}=O(\sqrt{n})$.
\end{abstract}

\section{INTRODUCTION}

Let $(\Omega, A, \operatorname{Pr})$ be a fixed probability space and let $\left\{g_{j}(\omega)\right\}_{j=1}^{n}$ be a sequence of independent identically distributed random variables defined on $\Omega$. Although there has been considerable attention given to algebraic and trigonometric polynomials with coefficients $g_{j}$ 's, very little is known about the behaviour of the random hyperbolic polynomial,

$$
P(x) \equiv P_{n}(x, \omega)=\sum_{j=1}^{n} g_{j}(\omega) \cosh j x
$$

Denote by $N_{K}(\alpha, \beta)$ the number of real roots of the equation $P(x)=K$ in the interval $(\alpha, \beta)$ and by $E N_{K}(\alpha, \beta)$ its expected value. The only literature that this author could find concerning $E N$ is a report by Bharucha-Reid and Sambandham [1, p. 110] on an unpublished result of Das [4], where it is stated that for $K=0$ and independent normally distributed coefficients with mean zero and variance one $E N_{0}(-\infty, \infty)$ is asymptotic to $(1 / \pi) \log n$. This is interesting as it shows that $E N_{0}$ for random hyperbolic polynomials does not correspond with that of the random algebraic polynomial

$$
F(x) \equiv F_{n}(x, \omega)=\sum_{j=1}^{n} g_{j}(\omega) x^{j}
$$

Received by the editors October 6, 1993.

1991 Mathematics Subject Classification. Primary 60H99; Secondary 42BXX.

Key words and phrases. Gaussian process, number of real roots, Kac-Rice formula, algebraic polynomials, trigonometric polynomials, fixed probability space. 
nor with that of the random trigonometric polynomial

$$
T(x)=T_{n}(x, \omega)=\sum_{j=1}^{n} g_{j}(\omega) \cos j x
$$

From Kac [7] or Wilkins [10] we know that for the algebraic polynomial (1.2), $E N_{0}(-\infty, \infty) \sim(2 / \pi) \log n$ is twice that of the hyperbolic case reported by Bharucha-Reid and Sambandham [1], while for the trigonometric case (1.3), $E N_{0}(0,2 \pi) \sim(2 n / \sqrt{3})$ (see Das [3] and Wilkins [9]). Therefore it is of special interest to establish for the hyperbolic case which of the known patterns, if any, $E N_{K}$, for $K \neq 0$, will follow. One can expect that, because of the similarity of order of $E N_{0}$, the $K$-level crossing would be similar to that of the algebraic case. In Farahmand [6] it is shown that $E N_{K}$ for the equation $F(x)=K$ is asymptotically reduced to $(1 / \pi) \log \left(n / K^{2}\right)$ in the interval $(-1,1)$ while its remains the same as $K=0$ in the interval $(-\infty, 1) \cup(1, \infty)$ as long as $K \equiv K_{n}=O(\sqrt{n})$. For the trigonometric equation $T(x)=K$, however, Farahmand [5] shows $E N_{K}(0,2 \pi)$ remains asymptotic to $(2 n / \sqrt{3})$. Our result here unexpectedly shows that the $K$-level crossing of the hyperbolic polynomial is similar to that of the trigonometric one. If one classifies the oscillation of different types of polynomials according to the behaviour of their real zeros, viz. the algebraic types with $E N_{0}=O(\log n)$ and the trigonometric types with $E N_{0}=O(n)$, it seems interesting to note that although random hyperbolic polynomials will fall into the first category, their properties of $K$-level crossings follow the second. We prove the following:

Theorem. For any sequence of constants $K_{n} \equiv K$ such that $\left\{K^{2} /(n \log n)\right\}$ tends to zero as $n$ tends to infinity the mathematical expectation of the number of real roots of the equation $P(x)=K$ satisfies

$$
E N_{K}(-\infty, \infty) \sim(1 / \pi) \log n
$$

\section{A FORMULA FOR THE EXPECTED NUMBER OF CROSSINGS}

Let

$$
\Phi(t)=(2 \pi)^{-1 / 2} \int_{-\infty}^{t} \exp \left(-y^{2} / 2\right) d t
$$

and

$$
\varphi(t)=d \Phi(t) / d t=(2 \pi)^{-1 / 2} \exp \left(-t^{2} / 2\right)
$$

then by using the expected number of level crossings given by Cramér and Leadbetter [2, p. 285] for our equation $P(x)-K=0$ we can obtain

$$
\begin{gathered}
E N(\alpha, \beta)=\int_{\alpha}^{\beta}(B / A)\left(1-C^{2} / A^{2} B^{2}\right)^{1 / 2} \varphi(-K / A) \\
\cdot[2 \varphi(\eta)+\eta\{2 \Phi(\eta)-1\}] d x
\end{gathered}
$$


where

$$
\begin{aligned}
& A^{2}=\operatorname{var}\{P(x)-K\}=\sum_{j=1}^{n} \cosh ^{2} j x, \\
& B^{2}=\operatorname{var}\left\{P^{\prime}(x)\right\}=\sum_{j=1}^{n} j^{2} \sinh ^{2} j x, \\
& C=\operatorname{cov}\left[\{P(x)-K\}, P^{\prime}(x)\right]=\sum_{j=1}^{n} j \sinh j x \cosh j x,
\end{aligned}
$$

and

$$
\eta=-C K / A\left(A^{2} B^{2}-C^{2}\right)^{1 / 2} \text {. }
$$

Let $\Delta^{2}=A^{2} B^{2}-C^{2}$ and $\operatorname{erf}(x)=\int_{0}^{x} \exp \left(-t^{2}\right) d t$; then from (2.1) we can write the extension of a formula obtained by Kac [7] and Rice [8] for the case of $K=0$ as

$$
E N(\alpha, \beta)=I_{1}(\alpha, \beta)+I_{2}(\alpha, \beta),
$$

where

$$
I_{1}(\alpha, \beta)=\int_{\alpha}^{\beta}\left(\Delta / \pi A^{2}\right) \exp \left(-B^{2} K^{2} / 2 \Delta^{2}\right) d x
$$

and

$$
I_{2}(\alpha, \beta)=\int_{\alpha}^{\beta}(\sqrt{2} / \pi) K C A^{-3} \exp \left(-K^{2} / 2 A^{2}\right) \operatorname{erf}(K C / A \Delta \sqrt{2}) d x .
$$

We remark that although we are interested in $x \in(-\infty, \infty)$ it is sufficient to restrict our attention to the number of real roots for positive $x$ only; since to each root of $P(x, \omega)=K$ in $(0, \infty)$ there corresponds a root of $P(x, \omega)=K$ in $(-\infty, 0)$, and conversely. Therefore $E N(-\infty, \infty)=2 E N(0, \infty)$. From (2.2)-(2.4) we obtain the following relations:

$$
\begin{gathered}
A^{2}=(2 n-1) / 4+\sinh (2 n+1) x / 4 \sinh x, \\
B^{2}=-n(n+1)(2 n+1) / 12+(2 n+1)^{2} \sinh (2 n+1) x / 16 \sinh x \\
-(2 n+1) \cosh (2 n+1) x \cosh x / 8 \sinh ^{2} x \\
+\left(2 \cosh ^{2} x / \sinh ^{2} x-1\right) \sinh (2 n+1) x / 16 \sinh x, \\
C=(2 n+1) \cosh (2 n+1) x / 8 \sinh x \\
-\sinh (2 n+1) x \cosh x / 8 \sinh ^{2} x,
\end{gathered}
$$

and therefore

$$
\begin{aligned}
\Delta^{2}= & \sinh ^{2}(2 n+1) x / 64 \sinh ^{4} x \\
& +(2 n-1) \sinh (2 n+1) x\left(1+\cosh ^{2} x\right) / 64 \sinh ^{3} x \\
& -\left(4 n^{2}-1\right) \cosh (2 n+1) x \cosh x / 32 \sinh ^{2} x \\
& +(2 n+1)\left(8 n^{2}-4 n-3\right) \sinh (2 n+1) x / 192 \sinh x \\
& -(2 n+1)^{2} / 64 \sinh ^{2} x-n(n+1)\left(4 n^{2}-1\right) / 48 .
\end{aligned}
$$




\section{PROOF OF THE THEOREM}

First we let $x$ be the interval $\left((\log n)^{1 / 2} / n, 1\right)$. As it turns out this interval will make the main contribution to the number of real roots. To find the dominant terms in (2.2)-(2.4) we observe that in this interval

$$
\operatorname{coth} x<e / x<e n(\log n)^{-1 / 2},
$$

and therefore the derivative of $f_{n, p}(x)=(\sinh n x)(\sinh x)^{-p}$ is positive for $p=1,2,3$, and for $n$ sufficiently large. Hence

$$
\begin{aligned}
f_{n, p}(x) & \geq \sinh \left\{(\log n)^{1 / 2}\right\}\left[\sinh \left\{(\log n)^{1 / 2} / n\right\}\right]^{-p} \\
& \geq \sinh \left\{(\log n)^{1 / 2}\right\}\left[(n / 4)(\log n)^{-1 / 2}\right]^{p} .
\end{aligned}
$$

Use has been made of the fact that $\sinh x \leq 4 x$ in $(0,1)$. Since $(\log n)^{1 / 2} / n$ is a decreasing function of $n,(3.1)$ will remain valid for $n$ replaced by $2 n+1$. Hence for all $n \geq 49$ and $p=1,2,3$ from (3.1) we can obtain

$$
f_{2 n+1, p}(x) \geq\left(n^{p} / 3.254\right)(\log n)^{-p / 2} \exp \left\{2(\log n)^{1 / 2}\right\} .
$$

Now from (2.8)-(2.11) and (3.2), for all sufficiently large $n$, and since $\sinh x \geq$ $x / 4$ in $(0,1)$ we can show

$$
\begin{aligned}
A^{2} & =[\{\sinh (2 n+1) x\} / 4 \sinh x]\left\{1+O(\log n)^{-1}\right\}, \\
B^{2} & =\left[(2 n+1)^{2}\{\sinh (2 n+1) x\} / 16 \sinh x\right]\left\{1+O(\log n)^{-1 / 2}\right\}, \\
C & =[(2 n+1)\{\cosh (2 n+1) x\} / 8 \sinh x]\left\{1+O(\log n)^{-1 / 2}\right\},
\end{aligned}
$$

and

$$
A^{2} B^{2}-C^{2}=\left[\{\sinh (2 n+1) x\} / 8 \sinh ^{2} x\right]^{2}\left\{1+O(\log n)^{-1}\right\} .
$$

In the following for $n$ sufficiently large we evaluate $I_{1}\left((\log n)^{1 / 2} / n, 1\right)$. To this end from (2.6) and (3.3)-(3.6) we have

$$
\begin{aligned}
I_{1}\left((\log n)^{1 / 2} / n, 1\right) & =(2 \pi)^{-1}\left[1+O(\log n)^{-1}\right] \\
& \cdot \int_{(\log n)^{1 / 2} / n}^{1}(\operatorname{csch} x) \exp \left\{-2 K^{2}(2 n+1)^{2} \sinh ^{3} x /\right. \\
= & (2 \pi)^{-1}\left[1+O(\log n)^{-1}\right] \int_{(\log n)^{1 / 2} / n}^{1}(\operatorname{csch} x) d x \\
& +O\left[K^{2}(2 n+1)^{2} \int_{(\log n)^{1 / 2} / n}^{1}\left\{\sinh ^{2} x / \sinh (2 n+1) x\right\} d x\right] .
\end{aligned}
$$

The first term appearing on the right-hand side of (3.7) can be evaluated as

$$
\begin{gathered}
(2 \pi)^{-1}\left[1+O(\log n)^{-1}\right]\left\{\log (\tanh 1 / 2)-\log \left[\tanh \left\{(\log n)^{1 / 2} / 2 n\right\}\right]\right\} \\
=(2 \pi)^{-1}\left[1+O(\log n)^{-1}\right]\left\{O(1)+\log \left\{n(\log n)^{-1 / 2}\right\}\right. \\
\left.-\log \left[1+O\left(n^{-2} \log n\right)\right]\right\} \\
=(2 \pi)^{-1}\{\log n-(1 / 2) \log (\log n)+O(1)\}
\end{gathered}
$$


Now we show that the second term appearing on the right-hand side of (3.7) is small compared with the value obtained in (3.8). To this end we write this term as

$$
\begin{aligned}
& O\left[K^{2}(2 n+1)^{2} \int_{(\log n)^{1 / 2} /(n+1 / 2)}^{1}\left\{x^{2} \operatorname{csch}(2 n+1) x\right\} d x\right] \\
& =O\left[K^{2}(2 n+1)^{-1} \int_{2(\log n)^{1 / 2}}^{2 n+1} u^{2} \operatorname{csch} u d u\right] \\
& =O\left[K^{2}(2 n+1)^{-1}\right] .
\end{aligned}
$$

Therefore, since $K=o(n \log n)^{1 / 2}$, from (3.7)-(3.9) we have $(2 \pi)^{-1} \log n$ as the asymptotic value for $I_{1}\left((\log n)^{1 / 2} / n, 1\right)$. Now we show $I_{1}\left(0,(\log n)^{1 / 2} / n\right)$ is small compared with this asymptotic value. For this range of $x$ the dominant term for $A^{2}, B^{2}$ and $C$ cannot be found. However, since from (2.8)

$$
A^{2}>\{\sinh (2 n+1) x\} / 4 \sinh x
$$

and since $u \operatorname{coth} u$ is an increasing function of $u$ we can have

$$
\begin{aligned}
\left(B^{2} / A^{2}\right) \leq & n(n+1)+(1 / 2) \operatorname{coth} x\{\operatorname{coth} x-(2 n+1) \operatorname{coth}(2 n+1) x\} \\
& -\{n(n+1)(2 n+1) \sinh x\} /\{3 \sinh (2 n+1) x\} \\
< & n(n+1)<(n+1 / 2)^{2} .
\end{aligned}
$$

Therefore for $x \geq 0$ and for all $n \geq 2$ from (3.10) we can obtain

$$
\left(\Delta / A^{2}\right) \leq(B / A)<n+1 / 2 \text {. }
$$

Therefore from (2.6), (3.11) and for all sufficiently large $n$ we can obtain

$$
I_{1}\left(0,(\log n)^{1 / 2} / n\right)=O\left((\log n)^{1 / 2}\right) .
$$

To estimate an upper limit for $I_{2}$ we note that since $C=(1 / 2) d\left(A^{2}\right) / d x$ from (2.7) we can write

$$
\begin{aligned}
I_{2}(0, \infty) & <(2 \pi)^{-1 / 2} \int_{0}^{\infty}|K C| A^{-3} d x \\
& =|K|(2 \pi)^{-1 / 2} \int_{n^{1 / 2}}^{\infty} A^{-2} d A \\
& =O\left(K / n^{1 / 2}\right) .
\end{aligned}
$$

Now it only remains to consider the case of $x \geq 1$ for $I_{1}$. From (2.8) and (2.11) and for sufficiently large $n$ we have

$$
\Delta^{2}<\sinh ^{2}(2 n+1) x / 16 \sinh ^{4} x
$$

and

$$
A^{2}>\sinh (2 n+1) x / 4 \sinh x .
$$

Therefore for all positive $x$,

$$
\left(\Delta / A^{2}\right)<\operatorname{csch} x .
$$

Hence from (2.6) and (3.14) we obtain

$$
\begin{aligned}
I_{1}(1, \infty) & <(\pi)^{-1} \int_{1}^{\infty}\left(\Delta / A^{2}\right) d x \\
& <(\pi)^{-1} \int_{1}^{\infty} \operatorname{csch} x d x=(\pi)^{1} \log \{\operatorname{coth}(1 / 2)\} .
\end{aligned}
$$


Finally from (3.8), (3.9), (3.12), (3.13), and (3.15) we have proof of the theorem.

\section{REMARK}

By looking at the proof it is apparent that although in the interval of $(-1,1)$ the hyperbolic polynomial has asymptotically as many roots as the algebraic polynomial, outside this interval, unlike the algebraic case, the hyperbolic polynomial does not possess any sizeable roots. Perhaps this is caused by (exponentially) fast increases (decreases) of the terms in $(-\infty,-1) \cup(1, \infty)$ which makes the cancellation in this type of polynomial difficult.

\section{ACKNOWLEDGMENT}

The author wishes to thank the referee for his detailed comments which improved the earlier version of this paper. It is a pleasure to thank Professor Sambandham for discussion on the earlier result of this paper.

\section{REFERENCES}

1. A. T. Bharucha-Reid and M. Sambandham, Random polynomials, Academic Press, New York, 1986.

2. H. Cramér and M. R. Leadbetter, Stationary and related stochastic processes, Wiley, New York, 1967.

3. M. Das, The average number of real zeros of a random trigonometric polynomial, Proc. Cambridge Philos. Soc. 64 (1968), 721-729.

4. $\ldots$, On the real zeros of a random polynomial with hyperbolic elements, Ph.D. dissertation, unpublished.

5. K. Farahmand, Level crossings of a random trigonometric polynomial, Proc. Amer. Math. Soc. 111 (1991), 551-557.

6. $\ldots$, Real zeros of random algebraic polynomial, Proc. Amer. Math. Soc. 113 (1991), 1077-1084.

7. M. Kac, On the average number of real roots of a random algebraic equation, Bull. Amer. Math. Soc. 49 (1943), 314-320.

8. S. O. Rice, Mathematical theory of random noise, Bell. System Tech. J. 25 (1945), 46-156.

9. J. E. Wilkins, Jr., An asymptotic expansion for the expected number of real zeros of a random polynomial, Proc. Amer. Math. Soc. 103 (1988), 1249-1258.

10. Soc. 111 (1991), 851-863.

Department of Mathematics, University of Ulster, Jordanstown, Co. Antrim BT37 OQB, UNITED KINGDOM 